\title{
Cardiovascular application of polyhedral oligomeric silsesquioxane nanomaterials: a glimpse into prospective horizons
}

This article was published in the following Dove Press journal:

International Journal of Nanomedicine

12 April 201 I

Number of times this article has been viewed

\author{
Hossein Ghanbari' \\ Achala de Mel' \\ Alexander M Seifalian ${ }^{1,2}$ \\ 'Division of Surgery and \\ Interventional Science, Centre for \\ Nanotechnology and Regenerative \\ Medicine, University College London, \\ London, England, UK; ${ }^{2}$ Royal Free \\ Hampstead NHS Trust Hospital, \\ London, England, UK
}

\begin{abstract}
Revolutionary advances in nanotechnology propose novel materials with superior properties for biomedical application. One of the most promising nanomaterials for biomedical application is polyhedral oligomeric silsesquioxane (POSS), an amazing nanocage consisting of an inner inorganic framework of silicon and oxygen atoms and an outer shell of organic groups. The unique properties of this nanoparticle has led to the development of a wide range of nanostructured copolymers with significantly enhanced properties including improved mechanical, chemical, and physical characteristics. Since POSS nanomaterials are highly biocompatible, biomedical application of POSS nanostructures has been intensely explored. One of the most promising areas of application of POSS nanomaterials is the development of cardiovascular implants. The incorporation of POSS into biocompatible polymers has resulted in advanced nanocomposite materials with improved hemocompatibility, antithrombogenicity, enhanced mechanical and surface properties, calcification resistance, and reduced inflammatory response, which make these materials the material of choice for cardiovascular implants. These highly versatile POSS derivatives have opened new horizons to the field of cardiovascular implant. Currently, application of POSS containing polymers in the development of new generation cardiovascular implants including heart valve prostheses, bypass grafts, and coronary stents is under intensive investigation, with encouraging outcomes.
\end{abstract}

Keywords: cardiovascular implants, nanocomposite, polymer

\section{Introduction}

The emergence of advanced novel biomaterials with improved properties, capable of being used in several biomedical applications has revolutionized the field of biomedical research in recent years. Nanomaterials are among intensively studied materials for a wide range of applications including biomedicine. Nanostructured composite materials are combinations of at least two constituent materials, a matrix (host) and a reinforcement component (guest, nanofiller). ${ }^{1}$ It is known that the properties of the materials change considerably when the size is significantly small, ie, within 1-100 nm size range. Since these materials have improved physical, chemical, and mechanical properties, they are significantly versatile for a wide range of applications.

Nanocomposite materials are sometimes considered to be either organic, inorganic, or hybrid in composition. ${ }^{2}$ Bone, abalone shells, and teeth are examples of nanocomposites in nature. Inspired by the enhanced properties of natural nanocomposites, there has been much interest in preparing synthetic nanocomposites and examining their properties for various potential applications. ${ }^{3,4}$ The method by which nanomaterials are synthesized is a crucial factor behind the behavior of nanocomposite materials.
Correspondence: Alexander M Seifalian Nanotechnology and Regenerative Medicine, University College London, Pond Street, London NW3 2QG, UK Tel +442078302901

Email a.seifalian@ucl.ac.uk 
For instance, in the case of polymer nanocomposite, the synthesis method along with the nature of nanofiller, the method of nanoreinforcement, and the type of interaction between polymeric and reinforcing components regulate the behavior of the polymer. ${ }^{5}$ In nanocomposites, nanofillers are able to intercalate between the layers of matrix (intercalated type) or be further dispersed uniformly throughout the matrix to form exfoliated nanocomposites, thus maximizing the surface area for component interaction. While intercalated nanocomposites have regular interlayers, exfoliated ones have larger interlayers (Figure 1).

Currently available synthetic nanocomposites can be classified into clay-, carbon-, metal- and glass-reinforced. ${ }^{6}$ Silicon and metal oxides (eg, $\mathrm{ZnO}$ and $\mathrm{TiO}_{2}$ ) are commonly used as nanofillers due to their substantial mechanical properties. ${ }^{7}$ These advanced materials have the potential to overcome the main restrictions and shortcomings of the standard synthetic materials owing to their enhanced properties. Among the most commonly studied nanoparticles for developing nanocomposite materials is the silsesquioxane family.

\section{Polyhedral oligomeric silsesquioxane (POSS)}

The chemical structure of the silsesquioxane family is defined as $\mathrm{R}_{\mathrm{n}} \mathrm{Si}_{\mathrm{n}} \mathrm{O}_{1.5 \mathrm{n}}$, which makes a structure consisting of an inner inorganic framework of silicon and oxygen atoms, externally covered by organic groups (R). R may either be hydrogen or an alkyl, alkene, aryl, and arylene group. Based on their molecular architecture, silsesquioxanes can be classified into two main categories of noncaged and caged nanostructure. The noncaged type silsesquioxane molecules can be further classified into random, ladder, and partial-cage nanostructure ${ }^{8}$ (Figure 2). Ladder like polysilsesquioxanes include poly(phenyl silsesquioxane), ${ }^{9}$ poly(methyl silsesquioxane),${ }^{10}$ and poly(hydridosilsesquioxane). ${ }^{11}$

Conventionally, cage-like silsesquioxanes appear as polyhedral oligosilsesquioxanes or polyhedral oligomeric silsesquioxanes (POSS). ${ }^{9}$ POSS is one of the molecules in the

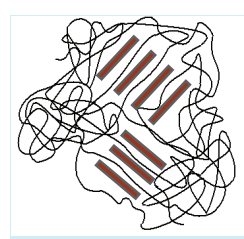

Intercalated

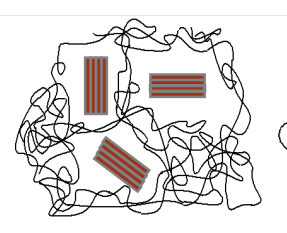

Phase separated

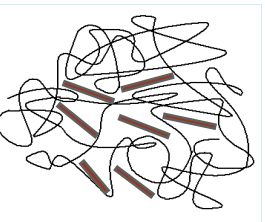

Exfoliated
Figure I Types of synthesized nanocomposites.
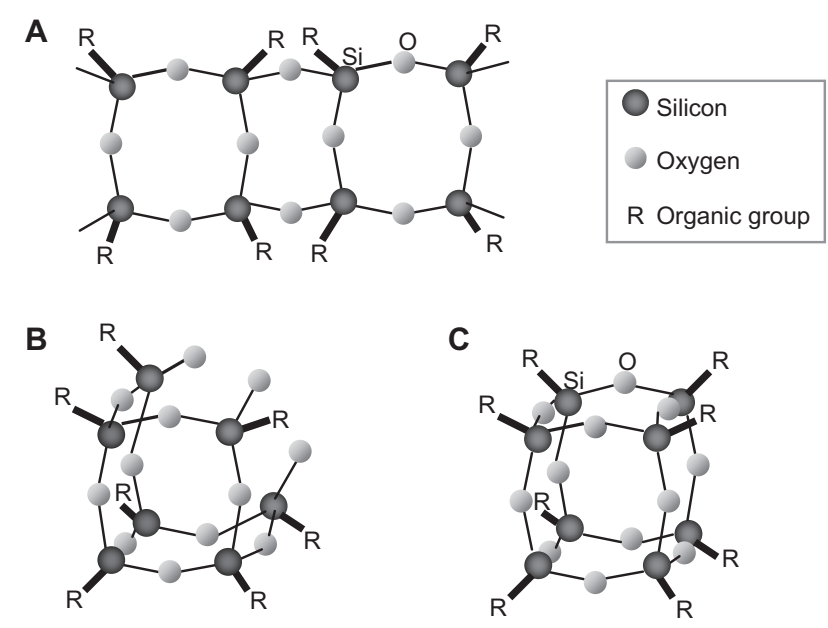

Figure 2 Different structures of silsesquioxanes: ladder structure (A), partial cage structure (B), and cage structure (C).

silsesquioxane family which has a regular three-dimensional (3D) shape (polyhedral = many-side, 3D) formed by a few units (oligomeric), each containing silsesquioxane. This is a structure consisting of an inner inorganic framework of molecular silica comprised of silicon atoms (n: 8) linked with oxygen atoms (n: 12) and an outer shell of organic groups (n: 8) that merged together to form a molecule. Hence, each silicon atom is bonded to three oxygen atoms and one organic group that may be inert or reactive. These are highly symmetric molecules with a nanoscopic size feature of approximately $1.5 \mathrm{~nm}$ in diameter including the (R) groups and can be considered as the smallest achievable silica particles. ${ }^{12}$

Based on the type of R group (eg, hydrogen, alkyl, alkylene, or aryl arylene), there are potentially an unlimited number of POSS types such as alkyls, olefins, alcohols, esters, anhydrides, acids, amines, imides, epoxies, thiols, sulfonates, fluoroalkyls, silanols, and siloxides. Researchers have shown that POSS cubes can be incorporated as building blocks ${ }^{13}$ to form hybrid inorganic-organic copolymers with improved thermal, mechanical, and physical properties. ${ }^{14,15}$ Physical and thermal properties improved by incorporation of POSS include, low dielectric constants $(\mathrm{K}),{ }^{16}$ increased glasstransition temperature, ${ }^{16-18}$ low coefficient of thermal expansion, thermal stability, and heat evolution. ${ }^{12,19}$ Improvement in the mechanical properties comprise tensile strength, ${ }^{17,18,20}$ viscosity, ${ }^{12,20,21}$ and enhanced viscoelastic properties. ${ }^{15,22,23}$ Further improvements have also been reported such as improved oxidation resistance, reduction of inflammability, oxygen permeability, and reduced inflammatory reactions, revealing the advantage of using these materials in various applications. ${ }^{17,18,20,24}$ Incorporation of POSS can change 
the surface properties including hydrophobicity, surface energy, and surface topography. Efficient surface coverage and stability to a variety of conditions are part of POSS derivative's features that make them attractive to be used as surface modification agents or other useful modifiers. ${ }^{25}$ Using POSS as a noncovalent modifier, a variety of $3 \mathrm{D}$ aggregates has been recently obtained from various nanoparticles such as palladium, magnetic, and gold nanoparticles. ${ }^{26-31}$ The unique characteristics of POSS nanoparticles offers diverse application potential in a wide range of areas, including the biomedical field, which is intensely under investigation.

\section{Biomedical applications of POSS}

The unique structure and superior properties of POSS allow them to be used in the structure of different polymers and copolymers developed for various biomedical applications. In particular, owing to their biocompatibility and ability to incorporate with different polymers, POSS nanostructures have been shown to offer high potential for several biomedical applications such as drug delivery, ${ }^{32,33}$ dental composites, ${ }^{34}$ biosensors, ${ }^{35}$ biomedical devices, ${ }^{36-38}$ and tissue engineering. ${ }^{39,40}$ Due to their inert nature and low inflammatory response, the framework of POSS, consisting of Si-O and Si-C, is very similar to silicone, which has been a preferable biomaterial since the 1960s, when it was introduced in breast implantation. ${ }^{41}$ Biocompatibility is the main characteristic of POSS, resulting from the increased surface energy in the foci of silicon-rich areas. Nontoxicity and cytocompatibility are other features of POSS, making them suitable for biomedical application. ${ }^{42-44}$ Hence, polymeric POSSbased nanocomposites have been largely studied by material scientists with the aim of using them in the biomedical field, in particular cardiovascular devices.

\section{Distinctive features of POSS nanocomposites for cardiovascular application}

Application of biomaterials in cardiovascular system and blood contacting devices necessitates specific characteristics such as blood compatibility and antithrombogenicity. To meet the essential requirements for these applications, we have developed a nanocomposite material by introducing POSS moieties into poly(carbonate-urea)urethane (POSS-PCU) as a pendent chain. Studies on its cytocompatibility, antithrombogenicity, and biostability have shown that this new nanocomposite polymer has unique characteristics for these applications. ${ }^{45}$ This nanocomposite can be bio-functionalized by modification of the surface to attract endothelial progenitor cells from the circulatory blood and become endothelialized to enhance bioand blood-compatibility of the cardiovascular devices made with this material (Figure 3). POSS-PCU has been characterized and assessed for biomedical applications in general and for cardiovascular application in particular, and the results of these studies reveal that POSS-PCU nanocomposite contains enhanced characteristics for these applications.

\section{Mechanical properties}

The development of cardiovascular devices such as prosthetic heart valves from polymeric materials requires a broad understanding of the basic mechanical and surface properties of the polymer. In a study, ${ }^{37}$ the mechanical properties of POSS-PCU, including tensile strength, tear strength, and hardness, were tested and compared with control (PCU). The surface properties were analyzed using contact angle measurement, and the resistance to platelet adhesion was also investigated. POSS-PCU (hardness $84 \pm 0.8$ Shore A) demonstrated significantly higher tensile strength $53.6 \pm 3.4$ and $55.9 \pm 3.9 \mathrm{~N} \mathrm{~mm}^{-2}$ at $25^{\circ} \mathrm{C}$ and $37^{\circ} \mathrm{C}$, respectively) than PCU $\left(33.8 \pm 2.1\right.$ and $\left.28.8 \pm 3.4 \mathrm{~N} \mathrm{~mm}^{-2}\right)$. Tensile strength and elongation at break of POSS-PCU was significantly higher than $\mathrm{PCU}$ at both $25^{\circ} \mathrm{C}$ and $37^{\circ} \mathrm{C}$. POSS-PCU showed a relatively low Young's modulus $(25.9 \pm 1.9$ and $26.2 \pm 2.0 \mathrm{~N} \mathrm{~mm}^{-2}$ ), which was significantly greater in comparison with control PCU $\left(9.1 \pm 0.9\right.$ and $\left.8.4 \pm 0.5 \mathrm{~N} \mathrm{~mm}^{-2}\right)$ at $25^{\circ} \mathrm{C}$ and $37^{\circ} \mathrm{C}$, respectively. There was no significant difference in tear strength between POSS-PCU and PCU at $25^{\circ} \mathrm{C}$. However, tear strength increased significantly (at $\left.37^{\circ} \mathrm{C}\right)$

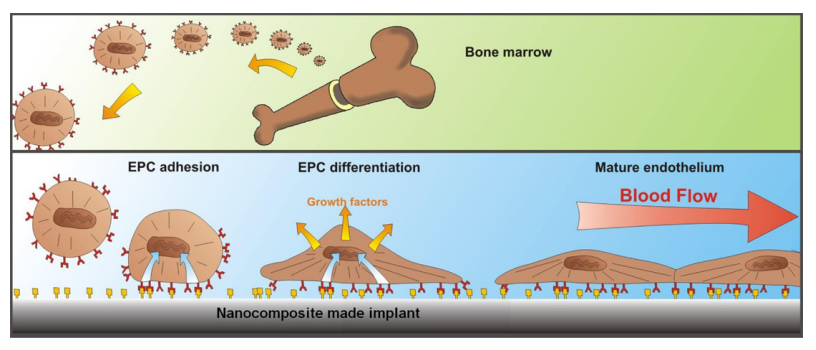

Figure 3 Biofunctionalization of the surface to enhance in situ endothelialization Biofunctionalized surface of the heart valve leaflets made from POSS-PCU nanocomposite can target several biological processes to promote in-situ endothelialization, by promoting the mobilization of EPC from the bone marrow, encouraging cell-specific (circulating EC, EPC, and stem cells) homing to the vascular graft site, providing cellspecific adhesion motifs on the vascular grafts (of a predetermined spatial concentration), and directing the behavior of the cells post-adhesion to rapidly form a mature fully functioning endothelium with self-repair capability.

Copyright $@$ 2008.American Chemical Society.Adapted with permission from De Mel A, Jell G, Stevens MM, Seifalian AM. Biofunctionalization of biomaterials for accelerated in situ endothelialization: a review. Biomacromolecules. 2008;9(I I):2969-2979. ${ }^{.5}$

Abbreviations: EC, endothelial cell; EPC, endothelial progenitor cells; POSS-PCU, polyhedral oligomeric silsesquioxane-poly(carbonate-urea)urethane. 
as the thickness increased from $100 \mu \mathrm{m}\left(51.0 \pm 3.3 \mathrm{~N} \mathrm{~mm}^{-1}\right)$ to $200 \mu \mathrm{m}\left(63 \pm 1.5 \mathrm{~N} \mathrm{~mm}^{-1}\right)$. The surface of POSS-PCU was significantly less hydrophilic than that of PCU. Thus, the mechanical characterization of the nanocomposite has proven to be an ideal candidate for the use in cardiovascular devices, in particular, synthetic heart valve leaflet application.

We have also developed and assessed a small porous caliber bypass graft made of POSS-PCU nanocomposite via an extrusion-phase-inversion manufacturing technique. Mechanical and elemental analysis (using energy-dispersive $\mathrm{x}$-ray analysis) showed a uniform distribution of POSS on the outer and inner surface of the graft with a favorable mechanical integrity for hemodynamic contacts. ${ }^{38}$

\section{Degradative resistance}

Initial expectations were that the presence of POSS nanocores in POSS-PCU material would hold together all the components of the polyurethane and thus prevent degradation thereby further improving upon the degradative resistance of polyurethanes. Polymer degradation of these nanocomposites was classified into three categories, namely physiological, oxidative, and hydrolytic. Fourier transform infrared (FTIR) analyses of the nanocomposite exposed to differing plasma protein fractions (PF I-IV) showed no difference in intensities of ' $\mathrm{Si}-\mathrm{O}$ ', 'C-O-C', 'NH-CO' (urea), and '(NHCO)O' (urethane) bonds for each bond wavelength as compared with the control. ${ }^{46}$ These results indicate that biological proteins have a negligible degradative effect on these POSS-PCU nanocomposites.

Thermal analysis of the most severely degraded POSSPCU nanocomposites showed that glass transition temperature $\left(\mathrm{T}_{\mathrm{g}}\right)$, an indicator of the soft phase of a polymer, did not vary significantly between the nondegraded and degraded samples, thus seconding the data on stress-strain analysis of the nanocomposite grafts. It was found that the addition of POSS-nanocores to PCU increased the final melting temperature of the nanocomposite by approximately $30^{\circ} \mathrm{C}$ while decreasing the $\mathrm{T}_{\mathrm{g}}$ by a further $10-12^{\circ} \mathrm{C} .{ }^{46}$ This paradox may be explained by the fact that nanocores prevent close packaging of polymer chains due to thermal rearranging, which in turn result in an increase in dynamic voids within the polymer. This extra space allows for earlier segmental mobility of the polymer chain and therefore lowering the $T_{g}$.

The increase in melting temperature $\left(\mathrm{T}_{\mathrm{m}}\right)$, and the lesser phase separation on the other hand, are attributable to the increased nanoscale interactions between its constituents, thus binding together the POSS-PCU nanocomposites. Furthermore, nanofilled-polyurethane (PU), unlike PCU, which shows three-phase melting, exhibited a single-phase melting process with higher temperatures at which softening occurs. In the t-butyl-degraded samples, the melt process was two-phased with lower softening temperatures. ${ }^{46}$ These results again signal that both hard and soft phases of the polymer integrate to a far greater extent than in PCU due to the nanoscale interaction of the POSS-nanocages with the soft phase.

\section{Biocompatibility and biostability}

To assess biocompatibility, sheets of POSS-PCU were implanted subcutaneously into the back of normal, healthy adult sheep for 36 months. After 36 months, the implants were removed from the animals under general anesthesia, and the surrounding tissue and capsule, if any, was taken for histopathological examination. The explanted nanocomposites exhibited no evidence of an inflammatory layer or capsule formation. ${ }^{47}$

Attenuated total reflectance (ATR)-FTIR analysis of the implanted 2\% POSS-PCU sheets showed that the 'Si-O' bonds at $1109 \mathrm{~cm}^{-1}$ wavelengths within the closed nanocage were intact in all the six samples of the nanocomposite and on both surface and bottom in different regions of the polymer. Scanning electron microscopy (SEM) analyses on the explanted nanocomposite samples showed no significant difference in its surface morphology as compared with the undegraded control POSS-PCU nanocomposite. ${ }^{47}$

The findings showed that POSS-PCU nanocomposites are ideal for use at the biological interface as tissue implants, biomedical devices, and even vascular grafts due to the very low levels of inflammation elicited by the host to it. This can be due to the intrinsic surface roughness of the polymer causing increased contact angle hysteresis and conformational change in adsorbed surface fibrinogen; a key initiator for foreign-body reactions.

\section{Endothelialization property}

Formation of an endothelial cell layer on the surface of cardiovascular implants enhances the bio- and hemocompatibility of these devices. In particular, the improvement of the endothelialization of the luminal surface of bypass grafts, potentially improve their patency rate and overall clinical outcomes. ${ }^{48}$ Studies have shown the clinical benefit of lining polytetrafluoroethylene (PTFE) vascular grafts with endothelial cells, particularly in lower limb arterial bypass grafts, ${ }^{49}$ unlike native, uncoated polyethylene terephthalate grafts. In an in-vitro study using human umbilical vein endothelial cells (HUVECs), we assessed whether this nanocomposite 
is safe and compatible with in vitro cell cultures. Apart from indicating its safety as a biomaterial at the cellular level, such information would also serve as a measure of its potential for developing bio-hybrid vascular grafts.

Compared with existing silicone copolymers, ${ }^{49-51}$ we found that the use of a POSS-based nanocomposite improves cell adhesion characteristics. As these nanocages occupy minimal volumes within the polymer, relatively greater surface areas of PU are available which allows for improved endothelialization. This would also confer a greater degree of polarity to the polymer which could explain its superior hydrophobicity to PCU.

These experiments also indicate that once adherent to its surface, endothelial cells (ECs) are also capable of proliferating manifold to form a confluent monolayer. While the PicoGreen ${ }^{\circledR}$ assay showed excellent proliferating characteristics of ECs on the polymer, light microscopic analysis revealed how this occurred. Prior to achieving cellular confluence, the ECs aligned in a reticular manner. The intervening areas were subsequently filled in to achieve cellular confluence. ${ }^{45}$ It has also been shown that exposing the EC-seeded POSS-PCU conduits to the low shear stress before being exposed to physiological shear stress significantly enhanced cell adherence rate. The findings approved potential benefits of the pre-seeding method followed by low shear stress and confirmed the potential of POSS-PCU for potential application in tissue-engineered cardiovascular devices. ${ }^{52}$

To enhance endothelialization potential, POSS-PCU can be biofunctionalized by modification of its surface using biofunctional peptides such as RGD (arginine-glycineaspartic acid), a functional part of an extracellular matrix component, fibronectin. In an in-vitro study, RGD-modified POSS-PCU sheets were assessed to evaluate cell adhesion and proliferation using peripheral blood mononuclear cells (PBMCs) containing endothelial progenitor cells (EPCs). The experimental data showed that the quality of cell adhesion and proliferation on the polymer was excellent. ${ }^{48}$ Qualitative data using SEM analysis clearly visualized the presence of optimal cell-polymer interactions with the formation of numerous filopodia at its surface, flattened ECs, and no rounded cells. This suggests that these ECs are capable of morphogenesis and have the ability to proliferate well. In another study, the RGD-modified POSS-PCU was used to develop a porous small diameter bypass graft and tested under static and pulsatile conditions. In this study PBMC containing EPCs isolated from adult healthy volunteers were cultured on nanocomposite graft and evaluated for the cell attachment, growth, and endothelialization using Alamar Blue assay, SEM, and immunostaining for endothelial cell markers, including CD34, CD31, and eNOS. This study showed that POSS-PCU has the bio-functionalization capability, and is able to provide cell growth and cell viability and have a great potential for rapid endothelialization from peripheral blood cells. ${ }^{53}$ Selective biofunctionalization using specific growth factors together with other surface modification techniques such as creating specific surface nanotopography can potentially enhance the affinity of the surface to ECs while minimizing possible attachment and proliferation of other cells such as smooth muscle cells or fibroblasts.

Natural regenerative capacity of the body can be utilized to induce endothelialization of cardiovascular devices. In a very recent study, we assessed the potential of in-situ endothelialization of a heart valve made from POSS-PCU nanocomposite. Peripheral blood mononuclear cells were separated using Ficoll-Paque centrifugation, with harvested EPCs purified using CD34 microbead labeling and magnetic-activated cell sorting (MACS). Cells were seeded onto 96-well plates coated with POSS-PCU or GRGDG/ GRGDG-LA modified POSS-PCU and PCU polymer controls for a period of 21 days. The cultured cells were studied under light, confocal, and SEM. In addition, fluorescence-activated cell sorting (FACS) was used to analyze cell surface markers including CD34, CD133, VEGFR2, CD144, CD31, and vWF in order to identify EPCs' potential proliferation and differentiation toward mature endothelial cells. Cell attachment and growth were observed in all POSS-PCU samples, with significantly higher activity than seen within the control polymers $(P<0.05)$. Microscopic examination revealed clonal expansion and morphological changes in cells seeded on POSS-PCU, GRGDG, and GRGDG-LA modified polymers. The frequency of triple-positive EPC (cells positive for CD34, CD133, and VEGFR2) was increased over time of culture, revealing the proliferation capacity of cultured EPCs. After one week of culture, SEM showed a mixed population of morphologically differentiated endothelial cells and EPCs. The cells expressed increasing levels of mature endothelial cell markers (CD31, vWF, CD144, and VEGFR2) over time with a confluent layer of EC on day 21 (Figure 4). These results support the self-endothelialization potential of cardiovascular implants made with the POSS-PCU polymer and the concept of the possibility of an in-vivo tissue engineering approach by the use of the natural regenerative capacity of EPCs and smart synthetic surfaces based on POSS-modified nanocomposite materials. 
A

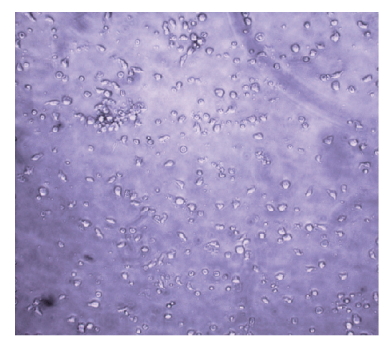

C

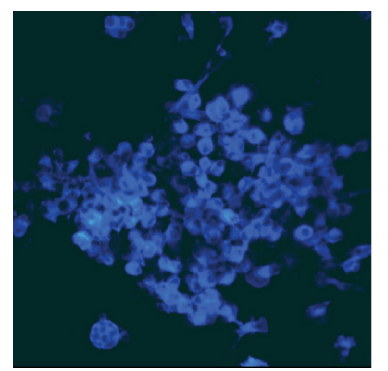

D

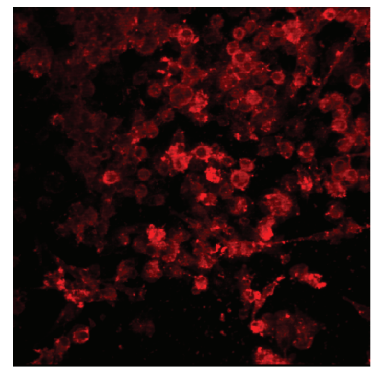

Figure 4 Morphological changes of isolated cells cultured on POSS-PCU nanocomposite polymer. Spindle-shaped morphology of early EPCs at day 7 (A) has been dominated by cobble stone-shaped features at day 21 (B) characteristics for the late EPCs or ECs; immunostaining of the cultured cells at day 14. Cells were stained for VWF (C) and VEGFR2 (D), showing positive expression of these cell surface markers on the cultured cells.

Abbreviations: EC, endothelial cell; EPC, endothelial progenitor cells; POSS-PCU, polyhedral oligomeric silsesquioxane-poly(carbonate-urea)urethane.

\section{Anti-thrombogenic potential}

To assess the antithrombogenic property of POSS, we used thromboelastography (TEG), a sensitive indicator of thrombogenicity. This was used as a screening test to ascertain the anti-thrombogenic properties of POSS-PCU. It was found that the polymer had significantly lower maximum amplitude values indicative of decreased platelet bonding strength as compared with control polystyrene and PCU. In addition, it was also found that the clots which formed on POSS-PCU were significantly unstable and lysed by 60 minutes compared with PCU or the control polystyrene polymer. ${ }^{54}$

Direct enzyme-linked-immunosorbent serologic assay (ELISA) fibrinogen adsorption analyses to the various polymers showed that there was a significantly decreased fibrinogen adsorption to the polyurethanes (POSS-PCU and PCU) as compared with PTFE. In the case of POSS-PCU, this may again be attributed to the effect of POSS groups on the PCU surface, which possess an unstable surface free energy and hence reduce both platelet and protein adsorption. Although we found no significant difference between POSS-PCU and PCU in terms of fibrinogen adsorption, Thromboelastography (TEG) analyses showed that the strength of the fibrin clot in POSS-PCU is much weaker as compared with PCU.
This indicated that while the amount of fibrinogen adsorbing to both polymers are similar, the binding strength is weaker in POSS-PCU compared with PCU alone.

Platelet adsorption onto casted sheets of POSS-PCU was significantly less than both PCU and PTFE sheets in vitro after 120 minutes in contact. There was significant difference in platelet adsorption by 120 minutes between POSS-PCU, PCU, and PTFE. In conjunction with the lower maximum amplitude values obtained on TEG, this suggests that POSSPCU has an anti-platelet effect by both repelling their surface adsorption and lowering the binding strength of platelets to the polymer which correspond to the poor adsorption characteristics exhibited towards fibrinogen as discussed above. ${ }^{47,54}$ The rate of platelet activation was also significantly lower in POSS-PCU samples. Direct ELISA analysis of the concentration of platelet factor 4 (PF4) released from platelets on the polymer surface was quantified. Significantly less concentration of PF4 was found from the supernatants of platelets incubated on POSS-PCU surface compared with the PCU control.

\section{Resistance to calcification and fatigue}

Calcification is a limiting factor in biomedical application of synthetic materials. In particular, it is considered as the main restricting factor of successful application of polymeric heart valves. ${ }^{55}$ Calcification also represents a major cause of failure of biological tissue heart valves. It is a complex phenomenon influenced by a number of biochemical and mechanical factors. In a study, ${ }^{36}$ the calcification-resistance efficacy and fatigue behavior of POSS-PCU were assessed by means of in-vitro testing. In particular, thin sheets of nanocomposite, glutaraldehyde fixed bovine pericardium (BP) and PU were exposed to a calcium solution into a specially designed invitro accelerated physiological pulsatile pressure system, for a period of 31 days and a total of $40 \times 10^{6}$ cycles. The samples were investigated for signs of calcification after exposure to calcium solution by means of $\mathrm{x}$-ray, microscopic, and chemical inspections. Mechanical and surface properties were also studied using stress strain behavior and surface morphology and hydrophobicity. Comparison showed that, in the experimental conditions, the level of calcification for the nanocomposite was considerably lower than for the fixed BP and PU samples (Figure 5). Also, mechanical properties were unchanged in POSS-PCU, while there was a significant deterioration in PU samples. Hydrophobicity significantly reduced in both POSS-PCU and PU samples. However, the POSS-PCU nanocomposite remained more hydrophobic than the PU sample. Less platelet adhered to the POSS-PCU compared with PU. ${ }^{36}$ 


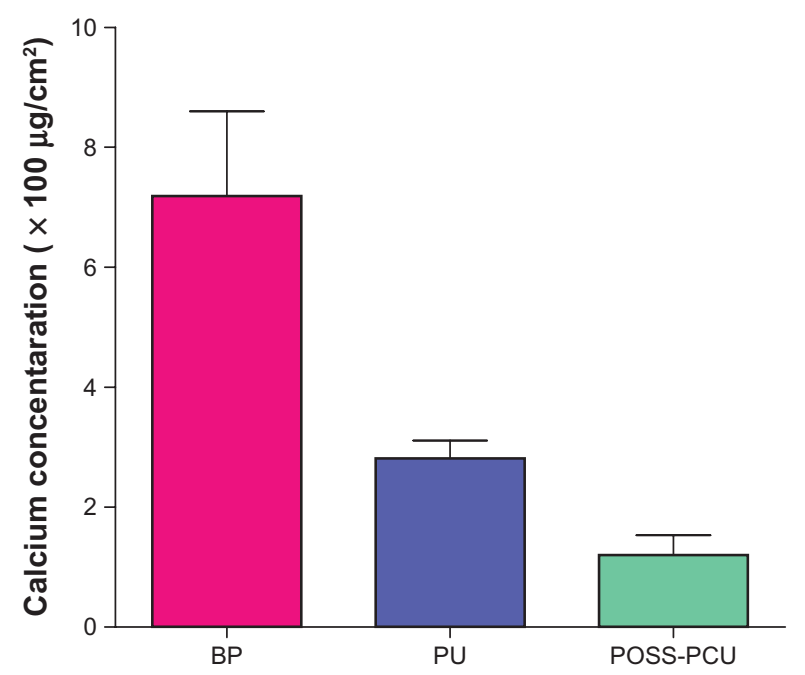

Figure 5 Chemical analysis of calcium deposition. Quantitative analysis of calcium deposition on the samples showed significantly reduced calcium deposition on POSS-PCU compared with BP $(P<0.00 \mathrm{I}, \mathrm{n}=5)$ and in comparison with PU $(P<0.05, \mathrm{n}=5)$.

Abbreviations: BP, bovine pericardium; POSS-PCU, polyhedral oligomeric silsesquioxane-poly(carbonate-urea)urethane; PU, polyurethane.

These results indicate that the use of this nanocomposite in synthetic leaflets heart valves may lead to potential advantages in terms of long-term performance and durability.

\section{Reduced inflammatory response}

Inflammatory response, which is mainly triggered by foreignbody reaction after transplantation, is also a determinant factor of biocompatibility and long-term durability of the biomedical implants. Inflammatory response is a very complex immunologic reaction involving various types of immune cells and immunomediators such as cytokines. All forms of implantation involve some degree of tissue injury, which will initiate two principal reactions, namely inflammation and the related response of repair. It is still incompletely understood; ie, which type and extent of inflammation favors optimal biocompatibility, and how precisely physicochemical properties of the biomaterials induce specific biological responses. However, it is well known that increased immune response and inflammatory reactions diversely affects the implant-host interaction, leading to unfavorable outcomes. In this in-vitro setting, we investigated potential inflammatory reactions induced by POSS-PCU compared with other polymers. Peripheral blood mononuclear cells were exposed to the polymeric disks for one week, and the activation of inflammatory cells was assessed by evaluation of cell surface markers and cytokine release. The study revealed that the level of pro-inflammatory cytokines, namely interleukin-1 (IL-1//IL-1F2) and tumor necrosis factor (TNF $\alpha / T N F S F 1 A)$ were significantly lower in the supernatant collected from POSS-PCU samples compared with the control PCU. This was in association with reduced expression of CD86 and CD69, which are leukocyte activation markers. The study revealed that incorporation of POSS into the PCU polymer can effectively reduce any possible inflammatory reaction resulting from the implantation of the biomedical devices made with these materials. It also enhances the hemocompatibility of cardiovascular devices by eliminating the well known effect of leukocyte activation on thrombosis formation.

\section{Development of cardiovascular implants using POSS}

The enhanced characteristics of POSS-PCU make it an ideal material of choice for the development of advanced cardiovascular implants. This polymer is currently being used in the fabrication of many biomedical devices, including synthetic leaflet heart valves, percutaneous valve prostheses, small and large diameter bypass graft, and coated stents, and stent grafts.

\section{Development of novel synthetic leaflet heart valve}

Heart valve replacements are among the most widely used cardiovascular devices and are in rising demand. Currently, clinically available devices are restricted to the mechanical and bioprosthetic valves which are associated with significant complications and drawbacks, mainly unsolved hemocompatibility problems of mechanical prostheses and poor durability of bioprosthetic counterparts. Synthetic leaflet heart valves based on newly emerged superior materials represent an attractive alternative to the existing prostheses, merging superior durability of mechanical valves and enhanced hemodynamic function of bioprosthetic valves. A novel synthetic leaflet heart valve has been in our lab using POSS-PCU nanocomposite as leaflet material ${ }^{37}$ (Figure 6). As POSS-PCU has been demonstrated to be safe, resistant to degradation, mechanically strong, able to be neo-endothelialized, biocompatible, and anti-thrombogenic, it can play a major role in the next generation of artificial heart valves. A novel polymeric heart valve design has been invented at University College London (UCL) which is based on a design strategy aimed at reducing the energy absorbed during the operating cycle, which results in high hydrodynamic performances and reduced stress levels. The enhanced hemodynamic performance of the UCL valve suggests that the proposed design is a good choice for developing a synthetic leaflet heart valve. This together with 
A

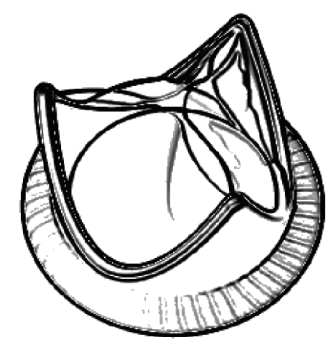

B

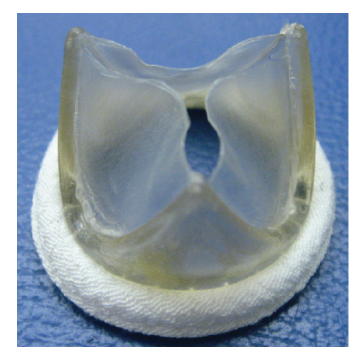

Figure 6 A) Trileaflet heart valve design (UCL design) with complex geometry and additional reflection on the leaflets to improve hemodynamic performance and durability. B) A valve prototype fabricated from POSS-PCU nanocomposite with a Dacron suture ring.

Abbreviations: POSS-PCU, polyhedral oligomeric silsesquioxane-poly(carbonateurea)urethane; UCL, University College London.

advanced POSS-PCU nanocomposite material with improved properties as the leaflet material indicates that UCL synthetic leaflet heart valve can be considered as a real breakthrough in the development of synthetic leaflet heart valve with potential successful clinical application, overcoming the limitations of the current prostheses.

\section{Percutaneous low profile valve prostheses}

Conventional open heart surgery for heart valve replacement consists of partial or complete sternotomy with extracorporeal circulation and cardioplegic cardiac arrest. This approach, in the case of the aortic valve replacement, is associated with high mortality rate (ranging from $10 \%$ to $50 \%$ ) in high-risk patients with severe co-morbidities such as left ventricular failure, concomitant coronary artery disease with prior bypass surgery, chronic obstructive pulmonary disease, and advanced age. ${ }^{56}$ Enormous development in interventional cardiology offers trans-catheter percutaneous techniques which could significantly reduce morbidity and mortality of valve repair or replacement, especially in high-risk elderly patients. Developing percutaneous valve replacement could extend treatment to those who are not currently considered for surgery and to those who are in early stages of valvular heart disease, and the treatment would avoid the onset of progressive ventricular dysfunction. ${ }^{57}$ Early results of experimental and clinical trials of percutaneous valve replacement are promising; however, the technique is in the early stage of its development with irrefutable potential problems which should be resolved. For instance, currently, modified bioprosthetic tissue valves are being used in minimally invasive valve replacement with concomitant complications. Advanced polymeric materials with improved properties can potentially be used as leaflet materials in percutaneous heart

valves. The use of synthetic materials enables the reduction of the leaflet thickness and therefore the overall profile of the valve. It also grants more consistent manufacturing techniques and eliminates the problem of tissue dehydration in the crimped state, which is one of the main complications of tissue valves. Using POSS-PCU nanocomposite, a novel aortic valve sui for trans-catheter aortic valve implantation is being developed by our group at UCL, aimed at overcoming the main limitations experienced with currently available percutaneous devices. The valve consists of three polymeric leaflets (POSS-PCU nanocomposite) attached to a self-expandable nitinol wire stent. The stent design provides a high expanded/collapsed diameter ratio and enhanced anchoring through sufficient radial and axial forces. The device is fully retrievable and repositionable, which is one of the main advantages of this device.

\section{Coronary artery bypass grafts and abdominal aortic aneurysms (AAA) grafts}

Coronary artery bypass surgery is one the most common cardiac surgeries worldwide. Currently, autologous blood vessels, such as the saphenous vein, are the conduits of choice for revascularisation. However, up to $30 \%$ of patients who require surgery do not have suitable or sufficient blood vessels to be used for transplantation due to vascular disease, amputation, or previous harvest. Furthermore, harvesting autologous vessels requires a second surgical procedure, with potential concomitant morbidies. Despite the critical need for synthetic small caliber grafts, success has been limited to the replacement of large caliber vessels, such as the aorta, where polyethylene terephthalate (PET) and expanded polytetrafluoroethylene (ePTFE) are the current industry standard biomaterials demonstrating impressive 3-year patency rates of over $90 \%{ }^{58}$ The replacement of small caliber vessels with synthetic grafts has been largely disappointing, with 2-year patency rates of $40 \%$ for the femoropopliteal position. ${ }^{59,60}$ Failure has been attributed primarily to thrombus formation, due to the inherent thrombogenicity of the synthetic surface, and intimal hyperplasia as a result of a mechanical mismatch between the elastic artery and rigid graft. ${ }^{61-63}$ A novel small diameter vascular graft with an internal diameter of $5 \mathrm{~mm}$ has been developed using POSS-PCU nanocomposite by utilization of thermally induced phase separation (TIPS) method which was shown to be compliant in nature, minimizing the mechanical mismatch between graft and artery (Figure 7). This graft has been studied in vitro and in vivo for hemocompatibility and reveals excellent patency rates in animal models and currently undergoing clinical trials. 
A

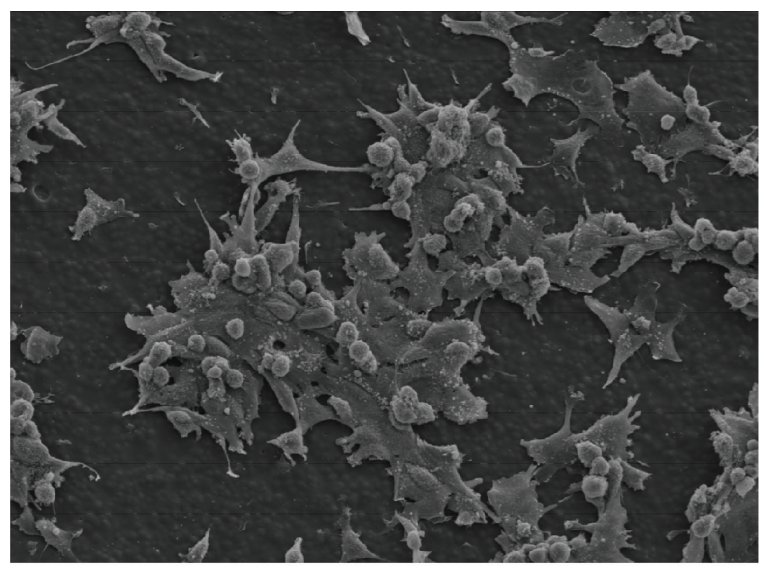

B

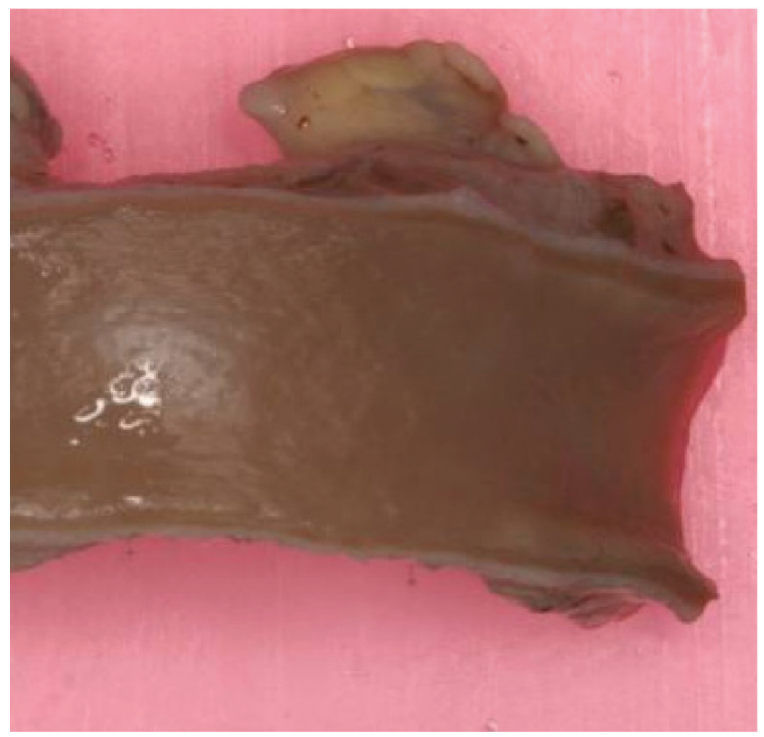

Figure 7 A) Scanning electron microscope pictures of endothelial cell adhesion morphology on POSS-PCU showing the presence of flat, spindle-shaped cells with numerous filopodia and the absence of cell retraction. This indicates the viability and proliferation of these cells on POSS-PCU at 48 hours (320x magnification). B) An explanted POSS-PCU bypass graft demonstrated to be endothelialized and patent after a 2 year implantation in a sheep model.

Abbreviation: POSS-PCU, polyhedral oligomeric silsesquioxane-poly(carbonateurea)urethane.

Along these lines, a large diameter abdominal aortic aneurism bypass graft is also being developed by the use of POSS-PCU and casting method. The graft is reinforced by self-expandable nitinol stent to be delivered via the vascular system using percutaneous approaches. Early results of the in-vitro tests of this innovative device are promising.

\section{Development of nanocomposite-coated coronary stents and stent grafts}

Application of POSS-PCU nanocomposite for coating coronary stents has also been explored by our group. Using electrohydrodynamic spraying or ultrasound atomization approaches, we have established the application of POSSPCU for the coating of metallic stents and demonstrated that this novel polymeric nanocomposite has the potential to be used for development of new generation stents with improved properties, especially in small-diameter stents including coronary artery stents. ${ }^{64}$ The coating would improve the hemocompatibility and patency rate of the commercially available stents which are prone to thrombosis and obstruction in long-term clinical use.

\section{Discussion}

Application of currently available cardiovascular implants is associated with serious complications and drawbacks, mainly due to poor hemocompatibility, inadequate durability, and lack of sufficient autologous replacements. Development of new generation implants based on advanced novel materials can potentially overcome the main problems in current clinical practice. The candidate material for this particular application should offer enhanced hemocompatibility and biostability to ensure improved long-term performance of the implant. POSS-PCU, as a paradigm POSS material, is a pioneering prototype of advanced novel materials which possesses superior hemocompatibility, biostability, antithrombogenicity, and calcification resistance, owing to its enhanced physicochemical properties and nanoreinforced structure and surface nanotopography. Its superior properties compared with conventional polymers are among the main advantages of using this material in the cardiovascular system. Early results of the development of small diameter bypass grafts and heart valve prototypes using this novel nanocomposite have been very promising. However, longterm in-vitro and in-vivo assessments are required in order to identify possible limitations and tangible advantages or disadvantages of using these new generation implants in real clinical practice. POSS nanocages have been incorporated into other polymeric systems, which have been explored for a wide range of biomedical applications, even though there is no report of cardiovascular applications of other POSS nanocomposites, to the best of our knowledge.

In-situ endothelialization of the implant surfaces is an interesting concept which can tremendously improve the hemocompatibility of these devices. This can be achieved by the use of state of the art surface modification techniques and the natural capacity of the human body. EPCs in the circulatory blood system hold great promises for this concept. Therefore, creating a smart surface to attract EPCs from peripheral blood, avoid excessive proliferation of smooth muscle cells and fibroblast, and retain a viable layer of 
functional endothelium is a challenging area which requires more research attention.

\section{Conclusion and future prospects}

The distinctive characteristics of POSS nanocomposites offer potential advantages over conventional polymers, in particular for cardiovascular applications. A novel POSS nanocomposite has been proposed for development of cardiovascular implants. The combination of POSS molecules with PCU polymer has resulted in a POSS-PCU nanocomposite with improved properties such as enhanced hemocompatibility, biostability, calcification resistance, and superior biomechanical features, which can be used in cardiovascular implants. With a nanocomposite suitable for cardiovascular application, attempts are now focused on development of new generation cardiovascular devices with improved hemocompatibility and long-term performance. As POSS nanomaterials are safe, resistant to degradation, compliant, allow neo-endothelialization, biocompatible and anti-thrombogenic, the probability of vascular occlusion is low. If successful in the long run, the clinical implications would be tremendous, as the superior properties of the new generation cardiovascular implants could overcome the complications and limitations of currently available devices. Projects on the development of percutaneous heart valves, coated stents and bypass grafts based on these POSS containing novel nanocomposites are currently active. Primary results of heart valve prototypes and coronary artery bypass grafts, fabricated from this novel nanocomposite, are very promising. Besides the mentioned properties, resistance to calcification, enhanced mechanical and surface properties, capability of grafting bioactive peptides to enhance attachment, proliferation, and differentiation of circulatory stem cells into endothelial cells are among other advantages of using these materials in the development of cardiovascular devices. By the emergence of new generation cardiovascular devices based on POSS nanomaterials, it is likely to overcome some of the major problems of current implants, saving lives and improving the quality of life of a significant number of patients.

\section{Acknowledgments}

The authors would like to thank Dr Rubin Kannan and Dr Gaetano Burriesci of UCL for useful comments and suggestions. The authors also acknowledge financial support of EPSRC, by grant to develop cardiovascular implants using POSS nanoparticle.

\section{Disclosure}

The authors report no conflicts of interest in this work.

\section{References}

1. Komarneni S. Nanocomposites. J Mater Chem. 1992;2(12): 1219-1230.

2. Huang HZ, Qiang Y, Yang XR. Morphology study of gold-chitosan nanocomposites. J Colloid Interface Sci. 2005;282(1):26-31.

3. Meng G, Cao A, Cheng JY, Ajayan PM. Carbon nanotubes grafted on silicon oxide nanowires. J Nanosci Nanotechnol. 2004;4(7): $712-715$.

4. Suhr J, Koratkar N, Keblinski P, Ajayan P. Viscoelasticity in carbon nanotube composites. Nat Mater. 2005;4(2):134-137.

5. Lee A, Lichtenhan JD. Viscoelastic responses of polyhedral oligosilsesquioxane reinforced epoxy systems. Macromolecules. 1998;31(15):4970-4974.

6. Liu YL, Hsu CY, Su YH, Lai JY. Chitosan-silica complex membranes from sulfonic acid functionalized silica nanoparticles for pervaporation dehydration of ethanol-water solutions. Biomacromolecules. 2005;6(1):368-373.

7. Yang $\mathrm{HG}$, Zeng HC. Synthetic architectures of TiO2/H2Ti5O11·H2O, $\mathrm{ZnO} / \mathrm{H} 2 \mathrm{Ti} 5 \mathrm{O} 11 \cdot \mathrm{H} 2 \mathrm{O}, \mathrm{ZnO} / \mathrm{TiO} 2 / \mathrm{H} 2 \mathrm{Ti} 5 \mathrm{O} 11 \cdot \mathrm{H} 2 \mathrm{O}$, and $\mathrm{ZnO} / \mathrm{TiO} 2$ nanocomposites. J Am Chem Soc. 2005;127(1):270-278.

8. Kannan RY, Salacinski HJ, Edirisinghe MJ, Hamilton G, Seifalian AM. Polyhedral oligomeric silsequioxane-polyurethane nanocomposite microvessels for an artificial capillary bed. Biomaterials. 2006;27(26):4618-4626.

9. Li GZ, Yamamoto T, Nozaki K, Hikosaka M. Crystallization of ladderlike polyphenylsilsesquioxane (PPSQ)/isotactic polystyrene (i-PS) blends. Polymer. 2001;42(20):8435-8441.

10. Maciel GE, Sullivan MJ, Sindorf DW. C-13 and Si-29 nuclear magnetic-resonance spectra of solid poly(methylsiloxane) polymers. Macromolecules. 1981;14(5):1607-1608.

11. Frye CL, Collins WT. Oligomeric silsequioxanes, (Hsio3/2)N. J Am Chem Soc. 1970;92(19):5586-5588.

12. Li GZ, Wang LC, Toghiani H, Daulton TL, Koyama K, Pittman CU. Viscoelastic and mechanical properties of epoxy/multifunctional polyhedral oligomeric silsesquioxane nanocomposites and epoxy/ ladderlike polyphenylsilsesquioxane blends. Macromolecules. 2001;34(25):8686-8693.

13. Draghi L, Resta S, Pirozzolo MG, Tanzi MC. Microspheres leaching for scaffold porosity control. J Mater Sci Mater Med. 2005; 16(12):1093-1097.

14. Sanchez C, Soler-Illia GJDA, Ribot F, Lalot T, Mayer CR, Cabuil V. Designed hybrid organic-inorganic nanocomposites from functional nanobuilding blocks. Chem Mater. 2001;13(10):3061-3083.

15. Hosseinkhani H, Hosseinkhani M, Kobayashi H. Design of tissueengineered nanoscaffold through self-assembly of peptide amphiphile. J Bioact Compat Polym. 2006;21(4):277-296.

16. Leu CM, Chang YT, Wei KH. Polyimide-side-chain tethered polyhedral oligomeric silsesquioxane nanocomposites for low-dielectric film applications. Chem Mater. 2003;15(19):3721-3727.

17. Fu BX, Gelfer MY, Hsiao BS, et al. Physical gelation in ethylene-propylene copolymer melts induced by polyhedral oligomeric silsesquioxane (POSS) molecules. Polymer. 2003;44(5): 1499-1506.

18. Haddad TS, Lichtenhan JD. Hybrid organic-inorganic thermoplastics: styryl-based polyhedral oligomeric silsesquioxane polymers. Macromolecules. 1996;29(22):7302-7304.

19. Huang JC, He CB, Xiao Y, Mya KY, Dai J, Siow YP. Polyimide/ POSS nanocomposites: interfacial interaction, thermal properties and mechanical properties. Polymer. 2003;44(16):4491-4499.

20. Oaten M, Choudhury NR. Silsesquioxane-urethane hybrid for thin film applications. Macromolecules. 2005;38(15):6392-6401. 
21. Fu BX, Namani M, Lee A. Influence of phenyl-trisilanol polyhedral silsesquioxane on properties of epoxy network glasses. Polymer. 2003;44(25):7739-7747.

22. Gupta A, Seifalian AM, Ahmad Z, Edirisinghe MJ, Winslet MC. Novel electrohydrodynamic printing of nanocomposite biopolymer scaffolds. J Bioact Compat Polym. 2007;22(3):265-280.

23. Langer R, Vacanti JP. Tissue engineering. Science. 1993;260(5110): 920-926.

24. Pellice SA, Fasce DP, Williams RJJ. Properties of epoxy networks derived from the reaction of diglycidyl ether of bisphenol A with polyhedral oligomeric silsesquioxanes bearing $\mathrm{OH}$-functionalized organic substituents. J Polym Sci Part B: Polym Phys. 2003;41(13): $1451-1461$.

25. Jeoung E, Carroll JB, Rotello VM. Surface modification via 'lock and key' specific self-assembly of polyhedral oligomeric silsequioxane (POSS) derivatives to modified gold surfaces. Chem Commun (Camb). 2002;(14):1510-1511.

26. He Y, Wang HF, Yan XP. Self-assembly of Mn-doped ZnS quantum dots/octa(3-aminopropyl)octasilsequioxane octahydrochloride nanohybrids for optosensing DNA. Chemistry. 2009;15(22): 5436-5440.

27. Frankamp BL, Fischer NO, Hong R, Srivastava S, Rotello VM. Surface modification using cubic silsesquioxane ligands. Facile synthesis of water-soluble metal oxide nanoparticles. Chem Mater. 2006;18(4):956-959.

28. Kannan RY, Salacinski HJ, Butler PE, Seifalian AM. Polyhedral oligomeric silsesquioxane nanocomposites: the next generation materia for biomedical applications. Acc Chem Res. 2005;38(11):879-884.

29. Naka K, Sato M, Chujo Y. Stabilized spherical aggregate of palladium nanoparticles prepared by reduction of palladium acetate in octa(3-aminopropyl)octasilsesquioxane as a rigid template. Langmuir. 2008;24(6):2719-2726.

30. Naka K, Itoh H, Chujo Y. Self-organization of spherical aggregates of palladium nanoparticles with a cubic silsesquioxane. Nano Lett. 2002; 2(11):1183-1186.

31. Carroll JB, Frankamp BL, Srivastava S, Rotello VM. Electrostatic selfassembly of structured gold nanoparticle/polyhedral oligomeric silsesquioxane (POSS) nanocomposites. J Mater Chem. 2004;14:690-694.

32. McCusker C, Carroll JB, Rotello VM. Cationic polyhedral oligomeric silsesquioxane (POSS) units as carriers for drug delivery processes. Chem Commun. 2005;8:996-998.

33. Tanaka K, Inafuku K, Naka K, Chujo Y. Enhancement of entrapping ability of dendrimers by a cubic silsesquioxane core. Org Biomol Chem. 2008;6(21):3899-3901.

34. Fong H, Dickens SH, Flaim GM. Evaluation of dental restorative composites containing polyhedral oligomeric silsesquioxane methacrylate. Dental Mater. 2005;21(6):520-529.

35. Zou QC, Yan QJ, Song GW, Zhang SL, Wu LM. Detection of DNA using cationic polyhedral oligomeric silsesquioxane nanoparticles as the probe by resonance light scattering technique. Biosens Bioelectron. 2007;22(7):1461-1465.

36. Ghanbari H, Kidane AG, Burriesci G, Ramesh B, Darbyshire A, Seifalian AM. The anti-calcification potential of a silsesquioxane nanocomposite polymer under in vitro conditions: potential material for synthetic leaflet heart valve. Acta Biomater. 2010;6(11):4249-4260.

37. Kidane AG, Burriesci G, Edirisinghe M, Ghanbari H, Bonhoeffer P, Seifalian AM. A novel nanocomposite polymer for development of synthetic heart valve leaflets. Acta Biomater. 2009;5(7):2409-2417.

38. Sarkar S, Burriesci G, Wojcik A, Aresti N, Hamilton G, Seifalian AM. Manufacture of small calibre quadruple lamina vascular bypass grafts using a novel automated extrusion-phase-inversion method and nanocomposite polymer. J Biomech. 2009;42(6):722-730.

39. Gupta A, Vara DS, Punshon G, Sales KM, Winslet MC, Seifalian AM. In vitro small intestinal epithelial cell growth on a nanocomposite polycaprolactone scaffold. Biotechnol Appl Biochem. 2009;54(4): $221-229$
40. Raghunath J, Zhang H, Edirisinghe MJ, Darbyshire A, Butler PE, Seifalian AM. A new biodegradable nanocomposite based on polyhedral oligomeric silsesquioxane nanocages: cytocompatibility and investigation into electrohydrodynamic jet fabrication techniques for tissue-engineered scaffolds. Biotechnol Appl Biochem. 2009;52(Pt 1):1-8.

41. Silver JH, Lin JC, Lim F, Tegoulia VA, Chaudhury MK, Cooper SL. Surface properties and hemocompatibility of alkyl-siloxane monolayers supported on silicone rubber: effect of alkyl chain length and ionic functionality. Biomaterials. 1999;20(17):1533-1543.

42. Cui D, Tian F, Ozkan CS, Wang M, Gao H. Effect of single wall carbon nanotubes on human HEK293 cells. Toxicol Lett. 2005;155(1):73-85.

43. Kim SK, Heo SJ, Koak JY, et al. A biocompatibility study of a reinforced acrylic-based hybrid denture composite resin with polyhedraloligosilsesquioxane. J Oral Rehabil. 2007;34(5):389-395.

44. Punshon G, Vara DS, Sales KM, Kidane AG, Salacinski HJ, Seifalian AM. Interactions between endothelial cells and a poly(carbonate-silsesquioxane-bridge-urea)urethane. Biomaterials. 2005;26(32):6271-6279.

45. Kannan RY, Salacinski HJ, Sales KM, Butler PE, Seifalian AM. The endothelialization of polyhedral oligomeric silsesquioxane nanocomposites: an in vitro study. Cell Biochem Biophys. 2006;45(2):129-136.

46. Kannan RY, Salacinski HJ, Odlyha M, Butler PE, Seifalian AM. The degradative resistance of polyhedral oligomeric silsesquioxane nanocore integrated polyurethanes: an in vitro study. Biomaterials. 2006;27(9):1971-1979.

47. Kannan RY, Salacinski HJ, Ghanavi JE, et al. Silsesquioxane nanocomposites as tissue implants. Plast Reconstr Surg. 2007;119(6):1653-1662.

48. Alobaid N, Salacinski HJ, Sales KM, et al. Nanocomposite containing bioactive peptides promote endothelialisation by circulating progenitor cells: an in vitro evaluation. Eur J Vasc Endovasc Surg. 2006;32(1):76-83.

49. Zilla P, Fasol R, Deutsch M, et al. Endothelial cell seeding of polytetrafluoroethylene vascular grafts in humans: a preliminary report. J Vasc Surg. 1987;6(6):535-541.

50. Ai H, Lvov YM, Mills DK, Jennings M, Alexander JS, Jones SA. Coating and selective deposition of nanofilm on silicone rubber for cell adhesion and growth. Cell Biochem Biophys. 2003;38(2):103-114.

51. Hesse Y, Kampmeier J, Lang GK, Baldysiak-Figiel A, Lang GE. Adherence and viability of porcine lens epithelial cells on three different IOL materials in vitro. Graefes Arch Clin Exp Ophthalmol. 2003;241(10):823-826.

52. Vara DS, Punshon G, Sales KM, Sarkar S, Hamilton G, Seifalian AM. Endothelial cell retention on a viscoelastic nanocomposite vascular conduit is improved by exposure to shear stress preconditioning prior to physiological flow. Artif Organs. 2008;32(12):978-981.

53. de Mel A, Punshon G, Ramesh B, et al. In situ endothelialization potential of a biofunctionalised nanocomposite biomaterial-based small diameter bypass graft. Biomed Mater Eng. 2009;19(4-5):317-331.

54. Kannan RY, Salacinski HJ, De Groot J, et al. The antithrombogenic potential of a polyhedral oligomeric silsesquioxane (POSS) nanocomposite. Biomacromolecules. 2006;7(1):215-223.

55. Ghanbari H, Viatge H, Kidane AG, Burriesci G, Tavakoli M, Seifalian AM. Polymeric heart valves: new materials, emerging hopes. Trends Biotechnol. 2009;27(6):359-367.

56. Grube E, Laborde JC, Zickmann B, et al. First report on a human percutaneous transluminal implantation of a self-expanding valve prosthesis for interventional treatment of aortic valve stenosis. Catheter Cardiovasc Interv. 2005;66(4):465-469.

57. Coats L, Bonhoeffer P. New percutaneous treatments for valve disease. Heart. 2007;93(5):639-644.

58. Polterauer P, Prager M, Holzenbein T, Karner J, Kretschmer G, Schemper M. Dacron versus polytetrafluoroethylene for Y-aortic bifurcation grafts: a six-year prospective, randomized trial. Surgery. 1992;111(6):626-633. 
59. Post S, Kraus T, Muller-Reinartz U, et al. Dacron vs polytetrafluoroethylene grafts for femoropopliteal bypass: a prospective randomised multicentre trial. Eur J Vasc Endovasc Surg. 2001;22(3):226-231.

60. Robinson BI, Fletcher JP. Fluoropolymer coated Dacron or polytetrafluoroethylene for femoropopliteal bypass grafting: a multicentre trial. ANZ J Surg. 2003;73(3):95-99.

61. Taylor RS, McFarland RJ, Cox MI. An investigation into the causes of failure of PTFE grafts. Eur J Vasc Surg. 1987;1(5):335-343.

62. Salzmann DL, Kleinert LB, Berman SS, Williams SK. Inflammation and neovascularization associated with clinically used vascular prosthetic materials. Cardiovasc Pathol. 1999;8(2):63-71.
63. Conte MS. The ideal small arterial substitute: a search for the Holy Grail? FASEB J. 1998;12(1):43-45.

64. Bakhshi R, Edirisinghe MJ, Darbyshire A, Ahmad Z, Seifalian AM. Electrohydrodynamic jetting behaviour of polyhedral oligomeric silsesquioxane nanocomposite. J Biomater Appl. 2009;23(4):293-309.

65. De Mel A, Jell G, Stevens MM, Seifalian AM. Biofunctionalization of biomaterials for accelerated in situ endothelialization: a review. Biomacromolecules. 2008;9(11):2969-2979.

\section{Publish your work in this journal}

The International Journal of Nanomedicine is an international, peerreviewed journal focusing on the application of nanotechnology in diagnostics, therapeutics, and drug delivery systems throughout the biomedical field. This journal is indexed on PubMed Central, MedLine, CAS, SciSearch $\AA$, Current Contents ${ }^{\circledR} /$ Clinical Medicine,
Journal Citation Reports/Science Edition, EMBase, Scopus and the Elsevier Bibliographic databases. The manuscript management system is completely online and includes a very quick and fair peer-review system, which is all easy to use. Visit http://www.dovepress.com/ testimonials.php to read real quotes from published authors. 